\title{
Numerical investigation of dense granular flow around horizontal tubes: qualification of CFD model with validated DEM model
}

\author{
Philipp Bartsch ${ }^{\mathrm{a}}$, Stefan Zunft ${ }^{\mathrm{b}}$ \\ ${ }^{a}$ Institute of Engineering Thermodynamics, German Aerospace Center, Pfaffenwaldring 38-40, Stuttgart, \\ Germany, Phone: 49-711-6862753, e-mail: philipp.bartsch@dlr.de \\ ${ }^{\mathrm{b}}$ Institute of Engineering Thermodynamics, German Aerospace Center, Pfaffenwaldring 38-40, Stuttgart, \\ Germany, Phone: 49-711-6862601, e-mail: stefan.zunft@dlr.de
}

\begin{abstract}
Granular material offer many advantages which qualify them for use as heat transfer medium and heat storage material in solar thermal power plants and industrial processes. Moving bed heat exchangers (MBHE) with horizontal tubes are favorable to extract thermal energy from hot granular materials. However, due to the complex flow phenomena and heat transport mechanisms in granular materials precise design tools for MBHE are lacking.

In previous studies we introduced a discrete particle model (DEM) to calculate the granular flow in MBHE and validated it by experiments. In this study we use this precise but computationally expensive DEM model to qualify a more efficient continuum model (CFD) which is being introduced in this work.

We compare the two models with regard to the granular flow speed along the tube surface and find deviations of $\left|\Delta u / u_{\mathrm{ref}}\right|<20 \%$ in the upper part of the tubes. Both models coherently predict the formation of a static area at the top of the tubes. The formation of the void area below the tubes is less accurately predicted, calling for further adaptations of the CFD model. The thus qualified CFD allows to study large heat exchanger geometries with substantially reduced computational effort.
\end{abstract}

\section{Introduction}

Granular materials have gained increasing attention as a promising candidate for application in thermal energy storage concepts. Being flowable, these materials can not only be used as a thermal energy storage medium but can also serve as a heat transfer fluid. Several works consider the utilization of granular materials as both the heat transfer medium and heat storage medium in solar thermal power (CSP) plants [1]. Advantages of such materials are their availability, thermal stability, non-toxicity and potentially low costs, especially when using natural stones like quartz sand. Studies have already been conducted identifying appropriate materials and their thermal and mechanical characteristics [2] [3]. Amongst others, sintered bauxite proppants and quartz sand have turned out to be promising candidates.

Among design alternatives, moving bed heat exchangers (MBHE) are favorable to extract thermal energy from hot granular materials. They offer a compact and robust design [4] and low parasitic loads. In a MBHE the granular material moves in vertical direction, driven by gravity. Immersed in the moving bed are heat-transferring surfaces such as horizontally arranged tubes.

An accurate prediction of the heat transfer rate at the tube surfaces requires detailed knowledge of the granular flow field around the tubes. Unfortunately, this information is difficult to access by measurement: On the one hand, experimental investigation of dense granular flows is difficult as an observation of the flow inside the bulk is very complex and costly, if at all possible. Optical measurement techniques, such as PIV, are restricted to the surface of the bulk and thus are likely to be influenced by boundary effects of the confining walls [5].

On the other hand, modelling of granular flows is a complex task as granular materials don't behave like Newtonian fluids: Discrete particle models, such as the discrete element method (DEM), offer detailed information about granular flows but are computationally expensive. In contrast, continuous model approaches are computationally light, but need adaptations through appropriate constitutive 
relations to properly account for the granular behavior of the flow. Such models are less accurate but offer to investigate large geometries.

Few works exist concerning continuum models for the slow, dense granular flow of a moving packed bed around horizontal tubes. Niegsch et al. [6] proposed a semi-empirical model which, however, neglects the influence of the flow geometry and is at least partially based on questionable assumptions. Lee et al. [7] used a FEM model to calculate the heat transfer between a moving bed and a single horizontal tube but showed no information at all regarding the calculated granular flow field. The CFD model of Baumann et al. [8] didn't capture the characteristic flow phenomena. Besides, several studies have been conducted for rapidly moving granular flows, such as fluidized beds (e.g. [9]) or rapid flows around obstacles [10]. Such models, however, are not suitable for the slow and friction-dominated granular flow of moving packed bed as will be further explained in section 3.1.

In a preceding study we introduced a discrete particle model (DEM) to calculate the granular flow around the horizontal tubes in a MBHE. The model has been validated on the basis of PIV measurements and proved to capture all relevant features of the flow [5]. In this paper we introduce an additional continuum model (CFD) and qualify it by comparing its results to those of the validated DEM model.

\section{Investigated geometry and granular flow pattern}

The investigated geometry is depicted in FIGURE 1 (left). It consists of a representative section of three rows of tubes arranged in a staggered manner. The granular material enters the geometry at the top. At the bottom we apply a fixed boundary condition for the outlet flow.

In the particle discrete model we simulate in $3 \mathrm{D}$ as quantities like the porosity and the coordination number ${ }^{1}$ of the moving bed are three-dimensional phenomena. In $z$-direction the geometry is 13 particle diameters thick and is confined by frictionless walls. In $x$-direction we use periodic boundaries.

The DEM results are averaged in space and time within stationary control volumes (for further information see [5]). The control volumes (CVs) keep a distance of three particle diameters from the front and rear walls (z-direction) to exclude boundary effects. For the evaluation of the velocity at the tube surface, the CVs are arranged in circular layers around the tube (CV size: $1.2 \mathrm{~mm}$ in radial direction, $2.5 \mathrm{~mm}$ in tangential direction, $7.0 \mathrm{~mm}$ in axial direction). The remaining simulation results are based on rectangular CVs with a size of $\Delta x=1.6 \mathrm{~mm}, \Delta y=1.6 \mathrm{~mm}$, and $\Delta z=7.0 \mathrm{~mm}$.

The continuum model simulations are in $2 \mathrm{D}$, transient and the motion of the interstitial fluid (air) is included. The model equations are given in the following section. The geometry is split along the vertical center line (see FIGURE 1 (left)), and only one half is simulated using symmetry boundary conditions in $x$-direction.

The boundary conditions for the continuum model are as follows: At the inlet we set the pressure of the fluid phase and the volume fraction for both phases. The velocity boundary condition at the tube walls deserves further explanation and is discussed in detail in section 3.2. At the outlet we set the gradient of the volume fraction normal to the boundary. Furthermore, a fixed outlet velocity is defined which is the same for both phases.

The mesh of the continuum model was refined until the simulation results converged. At the tube walls a local refinement was introduced to resolve the velocity gradient at the surface. The mesh size at the tube surface was approx. $0.6 \mathrm{~mm}$ in each spatial direction while in the rest of the geometry the mesh size was about $1.5 \mathrm{~mm}$.

The simulation was initialized with a uniform volume fraction $\left(\varepsilon_{\mathrm{s}}=0.56\right)$, a uniform velocity for both phases $\left(u_{\mathrm{y}}=u_{\mathrm{ref}}, u_{\mathrm{x}}=0\right)$ and ambient pressure. The simulation was run until a stationary state was reached.

\footnotetext{
${ }^{1}$ The coordination number is the number of contacts of a particle with its neighbors. It is of major importance for the heat transport in a packed bed [38] [30].
} 
On a standard four-core machine the DEM model took about 1.5 days to simulate a time interval of $9 \mathrm{~s}$. The CFD model takes about three to four hours depending on the initial conditions, using Ansys Fluent 18 CFD solver on a single core. The saving in computation time is expected to increase further when heat transfer is included in the simulation. In this case a time interval of about $30 \mathrm{~s}$ is estimated to reach a stationary state.

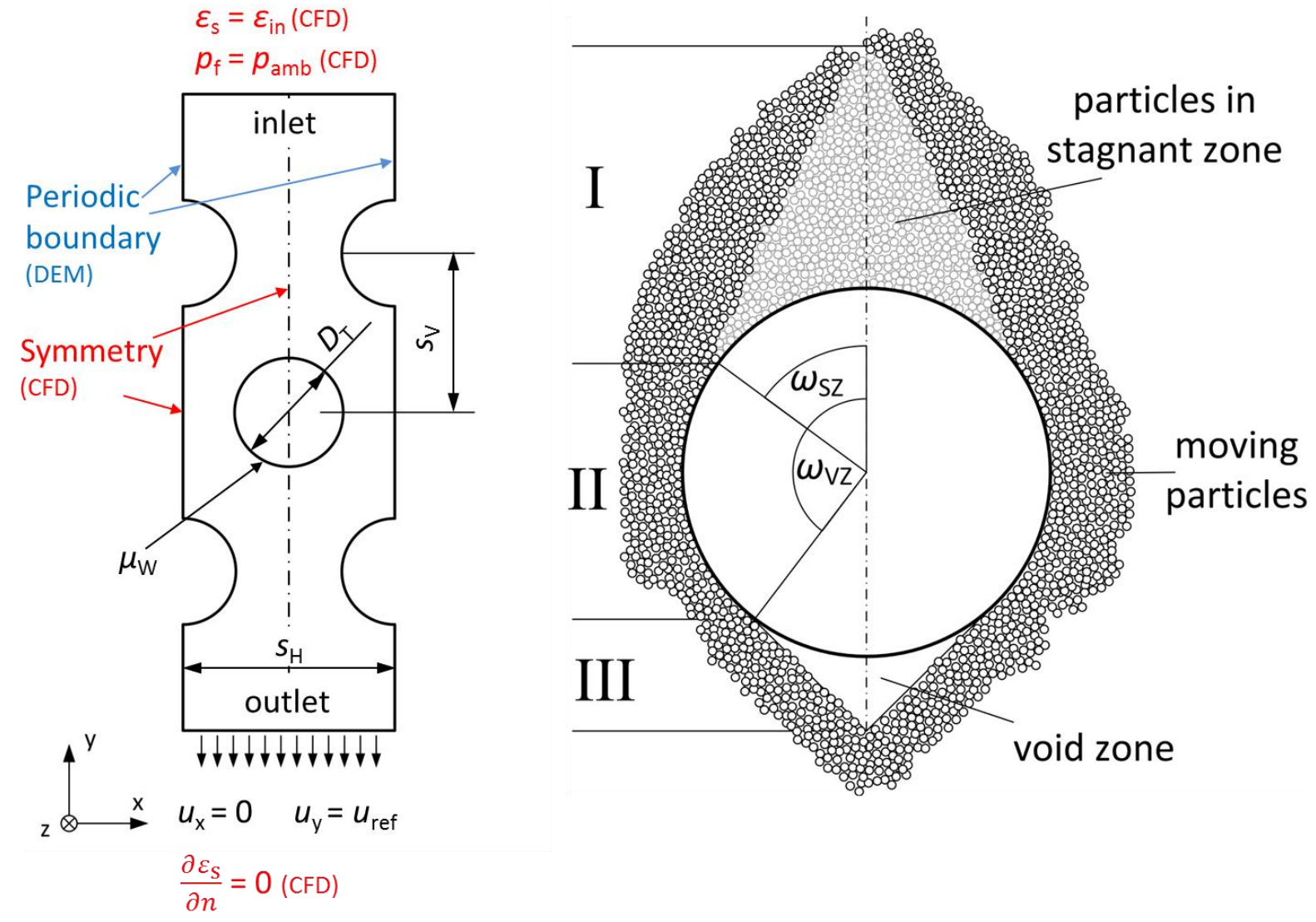

FIGURE 1. Simulation geometry (left). General granular flow pattern around horizontal tube as reported in literature (right).

Several researchers have investigated the granular flow pattern around horizontal tubes in the past [6] [11] [12]. The general flow pattern they reported is depicted in FIGURE 1 (right).

At the tube vertex a stagnant zone forms where the particles are approximately at rest. At a certain angle $\omega_{\mathrm{SZ}}$ the particles start to slide down the inclined surface of the tube. In the lower half of the tube at a certain angle $\omega_{\mathrm{VZ}}$ the particles detach from the tube surface and form a void area below the tube.

Niegsch et al. [6] estimated the values of $\omega_{\mathrm{SZ}}$ and $\omega_{\mathrm{VZ}}$ from basic considerations involving the inner friction coefficient of the bulk and the surface friction coefficient at the tube surface. By this means they estimated $\omega_{\mathrm{sZ}} \approx 60^{\circ}$ and $\omega_{\mathrm{VZ}} \approx 150^{\circ}$ for typical friction coefficients.

Likewise, they estimated the size of the stagnant area assuming a triangular shape. The resulting height of the stagnant area is $H_{\mathrm{SZ}} \approx 15 \mathrm{~mm}$ for typical material parameters (at a tube diameter of $27 \mathrm{~mm}$ as used in this work). These assumptions, however, have never been verified and it seems unlikely that the flow pattern should not depend on the flow geometry. In section 4, we compare them to the simulation results of this work.

\section{Continuum model equations}

The continuum model mainly follows the works of Srivastava et al. [13] and Schneiderbauer et al. [14]. The moving bed is modelled as two interpenetrating continua, one representing the granular 
phase and the other one representing the gas phase. Following the approach of Ishii [15], for both phases averaged continuity equations are solved as shown in eq. (1)-(3).

$$
\begin{gathered}
\frac{\partial}{\partial t} \varepsilon_{q} \rho_{q}+\boldsymbol{\nabla} \cdot\left(\varepsilon_{q} \rho_{q} \boldsymbol{u}_{q}\right)=0 \\
\frac{\partial}{\partial t}\left(\varepsilon_{g} \rho_{g} \boldsymbol{u}_{g}\right)+\boldsymbol{\nabla} \cdot\left(\varepsilon_{g} \rho_{g} \boldsymbol{u}_{g} \boldsymbol{u}_{g}\right)=-\varepsilon_{g} \boldsymbol{\nabla} p+\boldsymbol{\nabla} \cdot \varepsilon_{g} \boldsymbol{\tau}_{g}-\beta\left(\boldsymbol{u}_{g}-\boldsymbol{u}_{s}\right)+\varepsilon_{g} \rho_{g} \boldsymbol{g} \\
\frac{\partial}{\partial t}\left(\varepsilon_{s} \rho_{s} \boldsymbol{u}_{s}\right)+\boldsymbol{\nabla} \cdot\left(\varepsilon_{s} \rho_{s} \boldsymbol{u}_{s} \boldsymbol{u}_{s}\right)=-\varepsilon_{s} \boldsymbol{\nabla} p-\boldsymbol{\nabla} \cdot \boldsymbol{\sigma}_{s}+\beta\left(\boldsymbol{u}_{g}-\boldsymbol{u}_{s}\right)+\varepsilon_{s} \rho_{s} \boldsymbol{g}
\end{gathered}
$$

Here, the indices "s" and "g" denote the solid phase and the gas phase, respectively, and "q" is substitute for both phases. $\varepsilon$ is the volume fraction, $\boldsymbol{u}$ the velocity and $\rho$ the density of the corresponding phase. For the interphase drag coefficient $\beta$ a correlation of Gidaspow et al. [16] is used who applies the Ergun equation for high volume fractions:

$$
\begin{gathered}
\beta=\left\{\begin{array}{cl}
150 \frac{\varepsilon_{s}^{2} \mu_{g}}{\left(1-\varepsilon_{s}\right) d_{s}^{2}}+1,75 \frac{\varepsilon_{s} \rho_{g}\left|\boldsymbol{u}_{g}-\boldsymbol{u}_{s}\right|}{d_{s}} & \text { if } \varepsilon_{\mathrm{s}}>0.2 \\
0,75 C_{D} \frac{\varepsilon_{s}\left(1-\varepsilon_{s}\right) \rho_{g}\left|v_{g}-v_{s}\right|}{d_{p}}\left(1-\varepsilon_{s}\right)^{-2.65} & \text { if } \varepsilon_{\mathrm{s}} \leq 0.2
\end{array}\right. \\
C_{D}=\left\{\begin{array}{cl}
\frac{24}{R e_{g}}\left(1+0.15 R e_{g}^{0.687}\right) & \text { if }\left(1-\varepsilon_{\mathrm{s}}\right) R e_{\mathrm{g}}<1000 \\
0.44 & \text { if }\left(1-\varepsilon_{\mathrm{s}}\right) R e_{\mathrm{g}} \geq 1000
\end{array}\right.
\end{gathered}
$$

For the gas phase shear stress $\boldsymbol{\tau}_{\mathrm{g}}$ a Newtonian closure is used:

$$
\boldsymbol{\tau}_{g}=2 \mu_{g} \boldsymbol{D}_{g}
$$

where $\boldsymbol{D}=1 / 2\left(\boldsymbol{\nabla} \boldsymbol{u}+(\boldsymbol{\nabla u})^{T}\right)$ is the rate-of-deformation tensor.

\subsection{Frictional stress model}

What remains to be defined is the stress tensor of the solid phase $\sigma_{\mathrm{s}}$. In case of a strongly agitated bed, such as fluidized beds, particles interact by binary collisions. The stresses in such a rapidly moving granular flow are generally modelled using the so-called kinetic theory of granular fluids (KTGF) [17]. This approach follows the kinetic theory of gases assuming the particles to behave like gas molecules. In contrast to molecules, though, collisions between particles are highly inelastic, resulting in a strongly dissipative nature of "granular gases". As a consequence, granular gases can only be maintained by a constant supply of external energy, otherwise they collapse and form a densely packed particle assembly.

However, if the packing fraction is high $\left(\varepsilon_{\mathrm{s}}>0.4\right)$ as it is the case in moving beds, particles no longer interact by binary collisions but by enduring contacts with multiple neighbors. In this case KTGF is not valid anymore and a frictional stress model has to be applied. This kind of models is traditionally subject to soil mechanics and plasticity theory [18] and has been utilized to develop frictional stress closures for dense granular flows [19] [20] [21]. The bridging of both, the kinetic and the frictional flow regime is still a matter of research and different approaches have been proposed [22] [23] [24] [25].

As the packing fraction in a moving bed is close to the maximum packing fraction we assume that the granular flow is completely governed by frictional interaction between particles and we neglect the kinetic and collisional part. The frictional closure we use follows the approach of Jackson [26] and 
Tardos et al. [19] and has been used to model the discharging process of granular material from bins [13] [14] and hoppers [19]. The frictional stress is written in a compressible Newtonian form:

$$
\boldsymbol{\sigma}_{s}=\boldsymbol{\sigma}_{f r}=p_{f r} \boldsymbol{I}+2 \mu_{f r} \boldsymbol{S}_{s}
$$

Here, $\boldsymbol{S}_{s}=\boldsymbol{D}_{s}-\operatorname{tr}\left(\boldsymbol{D}_{s}\right)$ is the deviator of the strain rate tensor. The two parameters $p_{\mathrm{fr}}$ and $\mu_{\mathrm{fr}}$ are called frictional pressure and frictional viscosity, respectively, and are explained in more detail in the following paragraphs.

The frictional pressure accounts for the repulsive forces between grains and prevents the particle assembly from being compressed beyond a maximum packing fraction $\varepsilon_{\max }$. In soil mechanics $p_{\mathrm{fr}}$ is often combined with a yield function to describe the effect that a particle assembly may dilate or compact under shear movement. The theoretical framework in this context is called the critical state theory. A simplifying assumption of this theory is that the dilation and compaction effects are small and that the state of the material is close to the so-called critical state where it deforms without any volume change. This assumption has been proven to be justified for the flow in a discharging bin [13] and in hoppers [19]. Compared to this, the flow around a horizontal tube as it is being investigated in this work is more complex. Therefore the "critical-state-assumption" has been tested beforehand and it was found that the deviations from critical state were very small and hence the assumption is justified in this case as well. As a consequence $p_{\mathrm{fr}}$ is a function only of the packing fraction $\varepsilon_{\mathrm{s}}$. Several formulations for the critical state pressure have been proposed [27] and in this work we use the form of Johnson and Jackson [28]:

$$
p_{f r}=F \frac{\left(\varepsilon_{s}-\varepsilon_{\min }\right)^{r}}{\left(\varepsilon_{\max }-\varepsilon_{s}\right)^{s}}
$$

The parameter $\varepsilon_{\min }$ is the minimum packing fraction above which frictional interaction between particles occurs. If the packing fraction $\varepsilon_{\mathrm{s}}$ undercuts the minimum packing fraction $\varepsilon_{\min }$, the frictional pressure $p_{\text {fr }}$ is set to zero. The quantities in eq. (8) are set the same as in Bartsch et al. [8].

The second parameter in eq. (7), the frictional viscosity $\mu_{\mathrm{fr}}$, accounts for the shear stresses inside the granular material. One of the major challenges regarding the viscosity is that it should model the transition of a granular material from a "solid" (static) to a "flowing" state. This behavior manifests in granular flows by the formation of static areas where the particle aggregation behaves like one rigid body. The transition from a static to a flowing state takes place when the shear stress reaches a certain threshold. The simplest assumption for the threshold at which yielding occurs is a coulomb friction correlation $\left(\tau_{f r}=\mu_{i} \cdot p_{f r}\right)$, where $\mu_{\mathrm{i}}$ is an inner friction coefficient of the material. Based on this assumption Schaeffer [29] proposed a formulation for the granular viscosity:

$$
\mu_{f r}=\frac{p_{f r} \cdot \mu_{i}}{2\left|S_{s}\right|}
$$

The denominator of eq. (9) contains the Euclidian norm of the deviatoric part of the strain-rate tensor $\left|S_{s}\right|=\sqrt{S_{i j} S_{i j}}$. If the strain-rate (shear) approaches zero the viscosity diverges, which ensures the existence of a yield stress: the stress does not vanish when the flow stops [17].

The parameter $\mu_{\mathrm{i}}$ is an internal friction coefficient of the granular material. Schaeffer related $\mu_{\mathrm{i}}$ to the angle of internal friction $\phi_{\mathrm{i}}$ :

$$
\mu_{i}=\sqrt{2} \sin \left(\phi_{\mathrm{i}}\right)
$$

\subsection{Boundary conditions of granular phase at walls}

At a solid surface we have to decide whether the granular phase is slipping along the wall or whether it sticks to the wall. This is of particular interest with regard to the transition from a static to a flowing 
area. One might imagine a curved wall such as a tube surface where particles rest on top of the tube and start to slide along the circumference when a certain angle of inclination is reached.

In order to model this process we use a simple Coulomb friction law to define the granular velocity at a solid wall. Similar approaches have been used by used by Schneiderbauer et al. [14] and Johnson et al. [28].

The wall shear stress in slip-case is

$$
\tau_{s l}=\mu_{w} \cdot p_{f r},
$$

where $\mu_{\mathrm{w}}$ is a wall friction coefficient, $\tau_{\mathrm{w}}$ is the shear stress at the wall and $p_{\mathrm{fr}}$ is the normal stress. The wall shear stress is then compared to the viscose stress in tangential direction $\tau_{\mathrm{fr}, \text { tan }}$ inside the particle assembly close to the wall:

$$
\tau_{f r, t a n}=\mu_{f r}\left[\frac{d \boldsymbol{u}_{t a n}}{d \boldsymbol{n}}\right]
$$

The term $\left[\frac{d \boldsymbol{u}_{\tan }}{d \boldsymbol{n}}\right]$ is the gradient of the tangential part of the velocity $\boldsymbol{u}_{\mathrm{tan}}$ in the direction of the unit normal vector $\boldsymbol{n}$ of the wall. By comparing eq. (11) and eq. (12) the shear stress at the wall $\boldsymbol{\tau}_{\mathrm{W}}$ is determined:

$$
\boldsymbol{\tau}_{w}=-\frac{\boldsymbol{u}_{s}}{\left|\boldsymbol{u}_{s}\right|} \cdot\left\{\begin{array}{c}
\tau_{f r, t a n} \text { if } \tau_{s l}>\tau_{f r, t a n} \\
\tau_{s l} \text { if } \tau_{s l}<\tau_{f r, t a n}
\end{array}\right.
$$

\section{Simulation results}

In this section we compare the simulation results of the DEM and the CFD model applying the model parameters given in TABLE 1 . The friction parameters are chosen according to the properties of a typical bulk material such as quartz sand. In a follow-up paper we vary some of these parameters and examine their influence on the results.

TABLE 1. Simulation parameters.

\begin{tabular}{llll}
\hline Parameter & Symbol & Value & Unit \\
\hline Reference velocity (outlet) & $u_{\mathrm{ref}}$ & 5.0 & $\mathrm{~mm} / \mathrm{s}$ \\
Wall friction coefficient & $\mu_{\mathrm{w}}$ & 0.4 & - \\
Inner friction angle & $\phi_{\mathrm{i}}$ (continuum-model) & 30 & $\circ$ \\
Inter particle friction coefficient & $\mu_{\mathrm{pp}}(\mathrm{DEM})$ & 0.2 & - \\
Rolling friction coefficient & $\mu_{\mathrm{r}}$ (DEM) & 0.3 & - \\
Particle diameter & $d_{\mathrm{p}}$ (DEM) & 1.0 & $\mathrm{~mm}$ \\
Horizontal pitch & $s_{\mathrm{H}}$ & 57 & $\mathrm{~mm}$ \\
Vertical pitch & $s_{\mathrm{V}}$ & 50 & $\mathrm{~mm}$ \\
Tube diameter & $D_{\mathrm{T}}$ & 27 & $\mathrm{~mm}$ \\
\hline
\end{tabular}




\subsection{Flow field and packing fraction}

FIGURE 2 shows contours of the flow speed obtained from DEM (left) and CFD-model (right). The results of the continuum-model represent the velocity magnitude of the granular phase. The DEM results represent the particle velocities, averaged in space and time per control volume.

Both models coherently predict a stagnant area on top of the tubes. In both cases the stagnant zones are similar in size. Still, minor differences between both models are visible regarding the shape of the stagnant zone. The DEM results show a more curved shape whereas the continuum model yields a more tapered shape.

Niegsch et al. assumed that the stagnant area is confined by slip planes as they occur in triaxial tests with over-consolidated clays [30], separating resting from moving particles. Such a distinct transition from "static" to "flowing" is not observed in the simulations. Instead, both models show a continuous transition.

At the lateral sides of the tubes a maximum of the velocity magnitude occurs due to the constriction of the flow cross-section. The velocity magnitude decreases towards the tube surface due to wall friction but is not zero directly at the surface. The shear motion takes place in a narrow area near the wall which is typical for dense granular flows [31]. The maximum flow speed at the lateral sides of the tubes is slightly higher in the DEM simulation than in the CFD simulation.
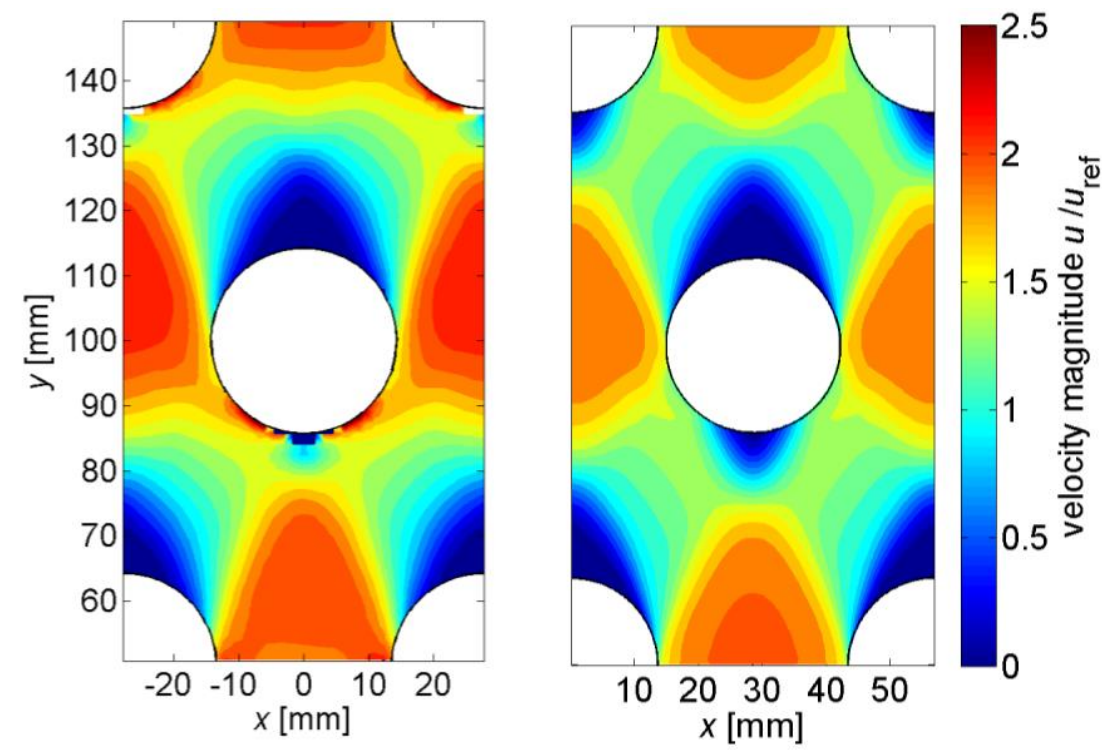

FIGURE 2. Contour plots of velocity magnitude at reference case. Comparison of DEM-model (left) and continuum-model (right).

In the lower half and underneath the tubes, we observe major deviations between the predicted flow fields. The DEM model yields a void zone where the packing fraction $\varepsilon_{\mathrm{s}}$ is zero (see FIGURE 3 (left)). The inclination of the flanks of this void zone is approximately the angle of repose, $\phi_{\mathrm{r}}$, of the material $\left(\phi_{\mathrm{i}} \approx \phi_{\mathrm{r}} \approx 30^{\circ}\right)$. In contrast to that, the continuum model yields only a slight decrease of the packing fraction below the tube (see FIGURE 3 (right)).

Similar observations have been made by other researchers in the past. For example, Nikolopoulos et al. [32] applied a similar continuum model to simulate a heap of granular material and compared the resulting repose angle to experimental data. They observed that the model can't produce a static angle of repose. Instead the material "deliquesces" at the free surface of the bulk.

A possible remedy for the problem could be the use of an elaborate yield function and a bulk viscosity to include viscous normal stresses in the model [32]. However, the distinct jump of packing fraction at the flanks of the void area is expected to remain difficult to be properly captured by a continuum model. Once a model is found which successfully can reproduce a static repose angle, it has to be supplemented by a description of the dilute and rapidly moving granular flow at the edges of the void zone to cover the full range of occurring volume fractions. 
Alternatively, the resulting decrease in heat transfer in this region might be accounted for by correction factors regarding the thermal resistance between the tube surface and the first layer of particles. Possible modifications of the model will be part of future investigations.

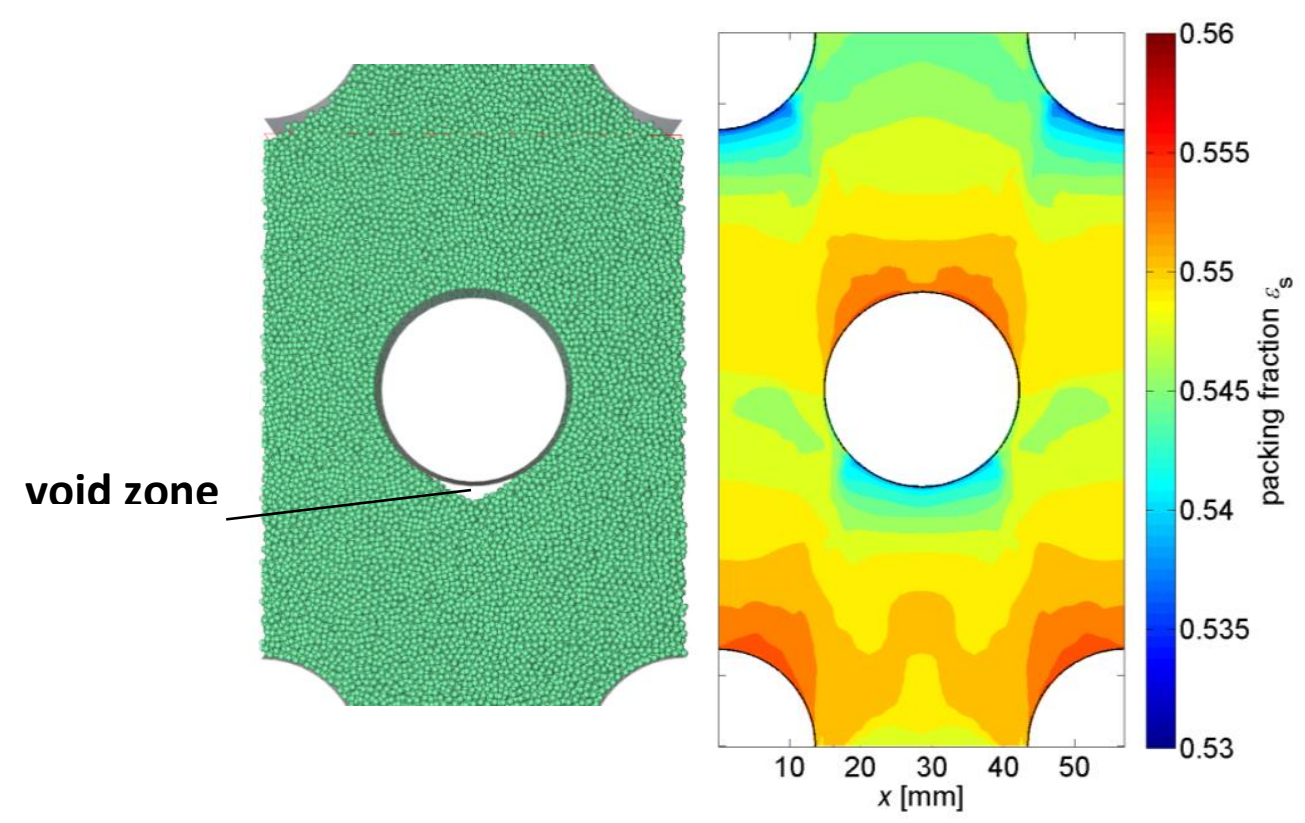

FIGURE 3. Particle assembly from DEM simulation with void area below the tube (left). Contour plot of packing fraction from CFD model (right).

\subsection{Velocity profile at the tube surface and above the tube}

The velocity profile in the direct vicinity of the tube surface has major impact on the heat transfer from the tube surface to the moving bulk. Therefore, an accurate modelling in this region is of particular importance.

FIGURE 4 (left) shows the velocity profile at the tube surface obtained from DEM and the CFD model. The velocity profile is that of the first layer of control volumes at the tube surface (see section 2), which corresponds to the first layer of particles). The results of the continuum model are obtained at a distance of $0.75 \mathrm{~mm}$ from the tube surface.

At the top of the tube $(\omega=0)$ the particles are at rest $\left(u / u_{\mathrm{ref}} \approx 0\right)$. Around a certain angle $\left(\omega_{\mathrm{Sz}}\right)$, the velocity starts to rise indicating the transition from the stagnant to the flowing regime. We estimate $\omega_{\text {Sz }}$ by inserting a tangent to the plots as shown in FIGURE 4 (left). Both models yield $\omega_{\mathrm{SZ}} \approx 45^{\circ}$. Compared to this, the value proposed by Niegsch et al. [6] $\left(\omega_{\mathrm{SZ}} \approx 60^{\circ}\right.$, cf. section 2$)$ appears rather high.

At the lateral sides of the tube, the velocity profile of the CFD simulation yields a maximum at $\omega \approx 90^{\circ}$ whereas the DEM simulation shows a local plateau. The results for the flow speed agree well in both models and the absolute deviation is $\left|\Delta u / u_{\mathrm{ref}}\right|<15 \%$.

In the lower part of the tube $\left(\omega>110^{\circ}\right)$ the DEM simulation shows a pronounced maximum of the velocity magnitude which does not occur in the CFD simulation. This maximum is associated with the formation of the void area (cf. FIGURE 3 (left)). The particles gradually lose the frictional contact with the tube surface and start to accelerate along the slope. This leads to elevated flow speeds in the lower half of the tube. As the effect is directly linked to the formation of the void area it is not apparent in the continuum model simulation which doesn't capture the void zone.

The phenomenon of high flow speeds in the lower half of the tubes has been observed in the past by Baumann et al. [12] using PIV measurements. They concluded that the static area of the subsequent tube-row grows into the free cross section between the tubes above. Their conclusion was supported by the size of the static areas they observed in their measurements. As seen above, though, the peak of 
flow speed doesn't originate from a reduced flow cross-section but from the formation of the void area. Recent studies have shown that PIV measurements are likely to be influenced by boundary effects in this case [5]. This finding illustrates the benefit of gaining information from inside the bulk through numerical simulations compared to measurements which are restricted to the surface of the bulk.
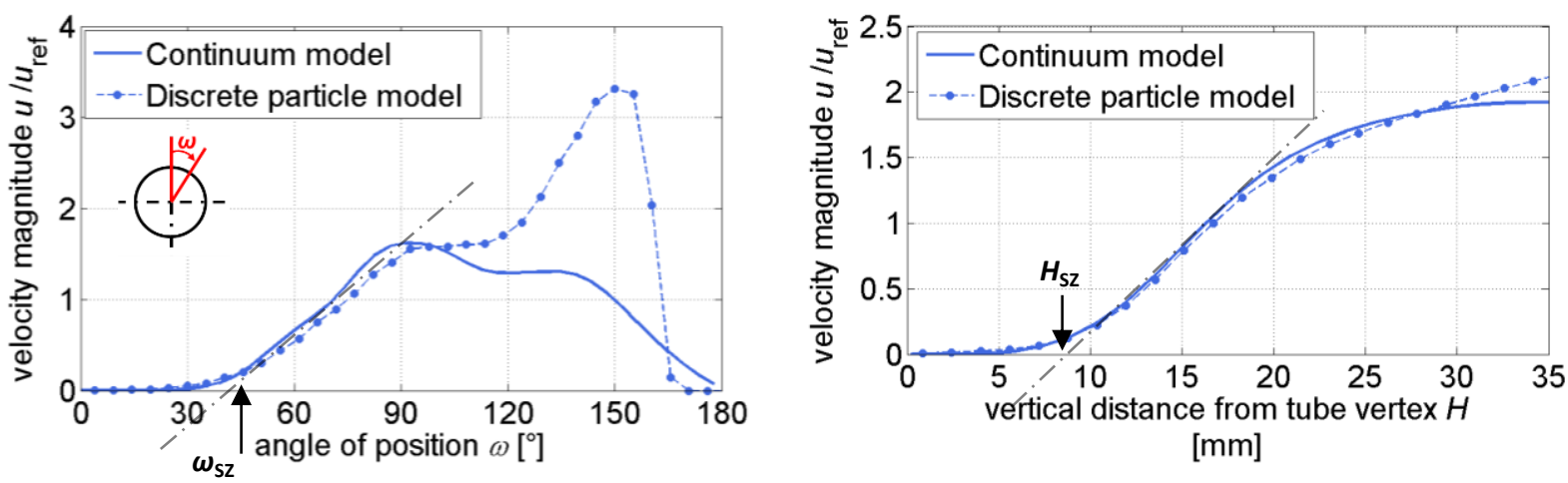

FIGURE 4. Granular flow speed along tube surface (left). Flow speed along vertical section above tube vertex (right).

FIGURE 4 (right) displays the velocity magnitude along the vertical center line above the tube vertex (see FIGURE 1 (right)). The results of DEM and continuum-model agree well and the absolute deviations are $\left|\Delta u / u_{\mathrm{ref}}\right|<15 \%$. We estimate the height of the static zone, $H_{\mathrm{SZ}}$, by inserting a tangent to the plots in FIGURE 4 (b) and taking the intersection with the x-axis. Both models yield $H_{\mathrm{SZ}} \approx 9 \mathrm{~mm}$ $\left(0.33 \cdot D_{\mathrm{T}}\right)$. This value is significantly smaller than the one estimated by Niegsch et al. [6] (cf. section 2). It remains to be investigated whether the size of the stagnant zone can be influenced by the heat exchanger geometry or other material and operating parameters.

\section{Summary and conclusions}

In this study we introduce a continuum model for the calculation of dense granular flows in moving bed heat exchangers with horizontal tubes. We qualify the model by comparing its results to those of a validated discrete particle model.

The comparison is based on selected contour plots and velocity profiles of the granular flow field. Particular attention is payed to the velocity profile at the tube surface.

Both models coherently yield a stagnant zone at the top of the tube. Furthermore, both models agree well with each other regarding the size and the extent of the stagnant area.

The velocity profiles at the tube surface show deviations of $\left|\Delta u / u_{\mathrm{ret}}\right|<15 \%$ in the upper half of the tube. In the lower half of the tube, major deviations between the two models are observed. The reason for these deviations is found in the formation of a void area below the tubes which is captured only by the DEM model.

Hence, the continuum model is considered valid in the upper half of the tube and in the realm of the stagnant area. In the realm of the void area below the tube the model needs to be modified.

Given these modifications, the model will be a valuable tool to evaluate the heat transfer rate in moving bed heat exchangers and will be used in upcoming design studies. 


\section{References}

[1] W. Wu et al., "Proof of concept test of a centrifugal particle receiver," Energy Procedia, vol. 49, pp. 560-568, 2014.

[2] T. Baumann, Wärmeauskopplung aus heißen Partikelschüttungen zur Dampferzeugung, Stuttgart: Stuttgart University, 2014.

[3] T. Baumann and S. Zunft, "Properties of granular materials as heat transfer and storage medium in CSP application," Solar Energy Materials and Solar Cells, vol. 143, pp. 38-47, 2015.

[4] A. Klein et al., "Schüttgutbewegung und Wärmetransport im Wanderbett - Teil 1," Chemie Ingenieur Technik, vol. 69, pp. 1130-1134, 1997.

[5] P. Bartsch and S. Zunft, "Granular flow around the horizontal tubes of a particle heat exchanger: DEM-simulation and experimental Comparison".submitted.

[6] J. Niegsch et al., "Heat transfer and flow of bulk solids in a moving bed," Chemical Engineering and Processing, vol. 33, pp. 73-89, 1994.

[7] W.-S. Lee, S.-K. Youn and S.-I. Park, "Finite element analysis of the flow and heat transfer of solid particles in moving beds," International Journal of Energy Research, vol. 22, pp. 1145$1155,1998$.

[8] P. Bartsch et al., "Granular Flow Field in Moving Bed Heat Exchangers: A Continuous Model Approach," Energy Procedia, vol. 99, pp. 72-79, 2016.

[9] M. J. V. Goldschmidt, R. Beetstra and J. Kuipers, "Hydrodynamic modelling of dense gasfluidised beds: Comparison of the kinetic theory of granular flow with 3D hard-sphere discrete particle simulations," Chemical Engineering Scinece, vol. 57, pp. 2059-2075, 2002.

[10] A. Morris, S. Pannala and C. Hrenya, "A conductive heat transfer model for particle flows over immersed surfaces," International Journal of Heat and Mass Transfer, vol. 89, pp. 1277-1289, 2015.

[11] H. Takeuchi, "Particles Flow Pattern and Local Heat Transfer Around Tube in Moving Bed," AIChE Journal, vol. 42, pp. 1621-1626, 1996.

[12] T. Baumann and S. Zunft, "Developement and performance assessment of a moving bed heat exchanger for solar central receiver power plants," Energy Procedia, vol. 69, pp. 748-757, 2015.

[13] A. Srivastava and S. Sundaresan, "Analysis of a frctional-kinetic model for gas-particle flow," Powder Technology, vol. 129, pp. 72-85, 2003.

[14] S. Schneiderbauer et al., "A comprehensive frictional-kinetic model for gas-particle flows: Analysis of fluidized and moving bed regimes," Chemical Engineering Science, vol. 80, pp. 279292, 2012.

[15] M. Ishii, Thermo-fluid dynamic theory of two-phase flow, Paris: Eyrolles, 1975.

[16] D. Gidaspow et al., "Hydrodynamics of circualting fluidized beds: Kinetic theory approach," in 7th Fluidization Conference, 1992.

[17] B. Andreotti et al., Granular Media - Between Fluid and Solid, Cambridge: Cambridge University Press, 2013.

[18] J. H. Atkinson and P. L. Bransby, The Mechanics of Soil: An Introduction to Critical State Soil Mechanics, England: McGraw-Hill, 1978.

[19] G. Tardos, "A fluid mechanistic approach to slow, frictional flow of powders," Powder Technology, vol. 92, pp. 61-74, 1997.

[20] B. Pitman and S. David, "Stability of time dependent compressible granular flow in two dimensions," Communications on pure and applied mathematics, vol. 40, pp. 421-447, 1987.

[21] M. Syamlal et al., MFIX Documentation Theory Guide, Morgantown: U.S. Dept. of Energy, Office of Fossil Energy, Tech. Note, 1993.

[22] I. S. Aranson and L. S. Tsimring, "Continuum theory of partially fluidized granular flows," Physical Review E, vol. 65, 2002.

[23] P. Jop et al., "A consititutive law for dense granular flows," nature, vol. 441, pp. 727-730, 2006. 
[24] S. B. Savage, "Analyses of slow high-concentration flows of granular materials," Journal of Fluid Mechanics, vol. 377, pp. 1-26, 1998.

[25] P. C. Johnson and R. Jackson, "Frictional-collisional constitutive relations for granular materials, with application to plane shearing," Journal of Fluid Mechanics, vol. 176, pp. 67-93, 1986.

[26] R. Jackson, "Some Mathematical and Physical Aspects of Continuum Models for the Motion of Granular Materials," Theory of Dispersed Multiphase Flow, pp. 291-337, 1983.

[27] B. G. M. van Wachem et al., "Comparative Analysis of CFD Models of Dense Gas-Solid Systems," AIChE Journal, vol. 47, pp. 1035-1051, 2001.

[28] P. C. Johnson et al., "Frictional-collisional equations of motion for particulate flows and their application to chutes," Journal of Fluid Mechanics, vol. 210, pp. 501-535, 1990.

[29] D. G. Schaeffer, "Instability in the Evolution Equations Describing Incompressible Granular Flow," Journal of Differential Equations, vol. 66, pp. 19-50, 1987.

[30] D. Schulze, Pulver und Schüttgüter, Berlin: Springer, 2009.

[31] M. Lätzel, From microscopic simulations towards a macroscopic description of granular media, Stuttgart University, 2003.

[32] A. Nikolopoulos, N. Nikolopoulos and N. Varveris et al., "Investigation of proper modeling of very dense granular flows in the recirculation system of CFBs," Particuology, 2012.

[33] W. Siu and S.-K. Lee, "Transient temperature computation of spheres in three-dimensional random packings," International Journal of Heat and mass Transfer, vol. 47, pp. 887-898, 2004.

[34] A. Slavin et al., "Theoretical model for the thermal conductivity of a packed bed of solid spheroids in the presence of a static gas, with no adjustable parameters at low pressure and temperature," International Journal of Heat and Mass Transfer, vol. 45, pp. 4151-4161, 2002.

[35] T. Baumann, S. Zunft and R. Tamme, "Moving Bed Heat Exchangers for Use With Heat Storage in Concentrating Solar Plants: A Multiphase Model," Heat Transfer Engineering, vol. 35, pp. 224-231, 2014.

[36] J. Niegsch et al., "Heat transfer and flow of bulk solids in a moving bed," Chemical Engineering and Processing, vol. 33, pp. 73-89, 1994. 